XIX.

Aus der psychiatrischen Klinik in Greifswald (Prof. A. Westphal.)

\title{
Beitrag zum Regenerationsvorgang in peripheren Nerven.
}

\author{
Voll \\ Dr. Max Lemke, \\ Assistent der Klinik. \\ (Hierzu Tafel X.)
}

In Band XXVIII dieses Archirs bat H. Gudden eine Arbeit über „Klinische und anatomische Beiträge zur Kenntniss der multiplen Alkoholneuritis nebst Bemerkungen über Regenerationsvorgänge im peripheren Nervensystem" veröffentlicht. An der Hand zahlreicher Abbildungen beschreibt er in seiner Arbeit eigenthümliche, breite, blasse, sich theilende Fasern, deren Bedeutung nicht ganz klar sei, indem er sagt: "In Osmiumzupfpräparaten von Fasern, besonders solchen, die dem Perineurium anliegen, gelingt es ausserordentlich häufig, eigenartige Fasern zu isoliren, die sich, was ihre Breite, Durchmesser und ibre Structur betrifft, sehr von den bisher bekannten Nervenfasern abheben... Die Myelinscheide ist hier möglicher Weise in chemischer Umwandlung begriffen, der Process entweder physiologisch, pathologisch oder regenerativ". Die letztere Annahme hat nach H. Gudden die meiste Wahrscheinlichkeit für sich.

Auf Anregung meines hochverehrten Chefs, Herrn Prof. Dr. A. Westphal, habe ich es unternommen, die bemerkenswerthen Befunde H. Gudden's, die bisher zu keinen weiteren Untersuchungen geführt haben; nachzuprüfen.

Dass solche Fasergebilde, wie Gudden sie gesehen hat, Schwierigkeiten in ihrer Deutung bereiten können, liegt auf der Hand, da sie zunächst als fremdartig imponiren, und man sie weder unter die de- 
und regenerative Form der Nervenfasern, noch unter sonstige bekannte Fasergebilde, wie Blutgefässe und Bindegewebe ohne weiteres unterbringen kann.

Schon S. Mayer erwähnt in seiner bekannten Arbeit über Vorgänge der Degeneration and Regeneration im anversehrten peripheren Nervensystem (Zeitschrift für Heilkunde 1881), blasser Strecken in den Nervenfasern. „... Sie können sehr mannigfaltig aussehn (durch versprengte Körnchen etc.) Tröpfchen, gelbliche Farbe und Grösse, sodass sie einer Kapillare ähnlich sehen ... ein faseriges Gebilde ist oft durch nichts weiteres als gewesene markhaltige Nervenfaser zu erkennen, als durch vereinzelte dunkele Körncben und Kerne ... wenn auch die feinkörnige Substanz bei der Resorption schwindet, so ist doch die Schwierigkeit gross, die Fasern als Nerven oder Bindegewebe zu erkennen."

Was die Theilungen der uns interessirenden Fasern anbelangt, so dürften diese, wemn auch ungewöhnlich, doch nicht ohne weiteres gegen deren nervöse Natur ins Feld zu führen sein.

So erwähnt Kölliker in seiner Gewebelehre Theilungen von Nervenfasern, besonders bei niederen Thieren, bei denen Markscheide wie Achsencylinder bedeutende Dickenzunahme erleiden, und die Aeste stets dünner als der Stamm sind:

Axel Key und Rezius (Studien in der Anatomie des Nervensystems und des Bindegewebes, 2. Hälfte, 1. Abtheilung, Tafel X) beschreiben und bilden Theilungen markhaltiger Nervenfasern aus Spinalnerven und dem Trigeminus des Hechtes ab.

Was die uns beschäftigenden Fasern betrifft, ist ausser den Theilungen, die Breite derselben, die durchsehnittlich das Doppelte bis Dreifache normaler Nervenfasern beträgt, ein auffallender Befund.

Ebenso wie Gudden fand auch ich die fraglichen Gebilde am häufigsten unter Fasern, welche dem Perineurium anlagen; da nun zu berücksichtigen ist, dass das Perineurium Träger der den Nerven ernährenden Blutgefässe jst, so.musste dieser Umstand von vomeherein auf die Wichtigkeit der Unterscheidung der sich theilenden Fasern von Blutgefässen, deren Schwierigkeit bereits Gudden herrorgehoben hat, hinweisen.

Bei meinen Lntersuchungen habe ich mich in erster Linie der Osmiumzupfpräparate bedient, aber auch Querschnitte sowie Marchi- und Saffraninfärbung angefertigt. Es standen mir dazu Nerven von 13 Fällen zur Verfügung (für die Veberlassung eines Theiles dieser Fälle bin ich Herrn Professor Grawitz zu bestem Danke verpflichtet) und zwar von Paralysen, Alkoholismus, Inanition, Nephritis und Dementia senilis. 
Da mich die mikroskopische Betrachtung der fraglichen Faseru allein nicht zum Ziele brachte, suchte ich auf experimentellem Wege der Lösung der mir gestellten Frage näher zu kommen und zwar durch Untersuchung der nach Durchschneidung peripberer Nerven auftretenden Degeneration. Ich experimentirte an Kaninchen im Alter von 5 and 8 Tagen, von 5 und 12 Wochen und von $3 / 4$ Jabren. An den Thieren resecirte ich circa $11 / 2 \mathrm{~cm}$ des Ischiadicus; nach 5 bis 7 Wochen tödtete ich die Thiere und injicirte ihnen ron der Aorta abdominalis aus eine Berlinerblaulossung von 5:100 Wasser, brachte dann die Extremitäten auf kurze Zeit in schwachen Alkohol, präparirte die Ischiadiei frei und fertigte davon die Zupfpräparate an.

Bei der Untersuchung der oben erwähnten 13 Fälle fand ich ausser bei den Nephritiden überall ziemlich typische Bilder der Fasern, wie sie Gudden 1. c. in zahlreichen vortrefflichen Abbildungen wiedergegeben hat. Daneben sah ich besonders bei Dementia senilis aber auch bei Paralysen und Inanition in Uebereinstimmung mit den bekannten Befunden von Oppenheim und Siemerling (Dieses Archiv Bd. XVIII) gewöhnliche degenerirte Nervenfasern in wechselnder Menge. Von den in Rede stebenden Fasern giebt besser als Worte es können, Fig. 1 ein Bild, das aus dem N. medianus einer 32 jührigen Melancholica, die an Inanition zu Grunde ging, stammt. Wir sehen hier ein hellgelbes faseriges Gebilde von etwa dreifacher Breite normaler Nervenfasern; in demselben lange, ovale zum Theil rundliche Kerne, die eine feine Körnelung zeigen, z. Th. grössere, tief schwarze Köruchen enthalten; neben der Faser und in ibrem unteren Theil am Rande derselben hellgelbe glänzende rundliche Gebilde von der Grösse der Blutkörperchen; ausserdem zeigt die Faser einen axialen dunkeln Faden und oine Einschnürung. Man kann angesichts dieses Bildes vielleicht mit Gudden an eine regenerirte Nervenfaser denken, trotzdem manches dagegen spricht: die auffallige Breite, der vollkommene Mangel an grösseren Markresiduen (im ganzen Faserverlauf waren solche nicht zu finden) und die immerhin doch etwas sonderbare Configuration; anch die eigenthümliche Einschnürung passt nicht recht in das gewohnte Bild; ebenso kann der axiale Faden nichts beweisen, da ja der Achsencylinder bei Osmiumzupfpräparaten nicht in electiver Weise gefärbt wird. Als Blutgefäss lässt sich die Faser hier auch nicht erkennen; jedoch bin ich auf Grund meiner weiteren Untersuchungen gezwungen, dieselbe für ein solches zu halten. Den Beweis der grössten Wahrscheinlichkeit hierfür werde ich im Folgenden zu erbringen sucben.

Betrachten wir Fig. 2 aus dem N. peroneus eines Paralytikers und Fig. 3 aus dem Peron, einer an Dementia senilis zu Grunde gegangenen 
Frau. Es sind sozusagen dieselben Bilder wie Fig. 1, deren nähere Beschreibung sich also eribbrigt. Hier nun ist der Beweis, dass wir es mit Capillaren zu thun haben, leicht, da Fig. 2 a bezw. 3 a Stücke derselben Fasem an entfernteren Stellen geben, und dieselben sich hier ohne Weiteres als Blutgefässe demonstriren. Oft findet man aber bei einer solchen fraglichen Faser in deren ganzem Verlaufe keine einzige Stelle, die ihre wahre Natur verriethe. Thabei kam mir nun das Experiment zu Hilfe.

In Fig. 5 ( 8 Tage altes Thier, nach 6 Wochen injicirt), sehen wir eine Faser, die in Allem den vorigen gleicht und ansserdem einen Ansatz zur Theilung zeigt. In der Achse findet sich die blaue Injectionsmasse, die das Gebilde zweifellos als Gefäss erkennen lässt. Ieh fand die lnjectionsmasse in kleinen blanen Partikelchen an den verschiedensten Stellen der Faser zerstreut, immer in der Achse derselben liegend und zwar fast bei allen Osmium-Zupfpräparaten, die von injicirten Thieren stammten. Theils konnte ich sich theilende Fasern beobachten, wie in Fig. $7(3 / 4$ Jahr altes Thier), theils waren Theilungen nicht zu entdecken; vergl. Fig. 6 und 8 von demselben Thier. In letzterer Faser sind einige sich mit Osminm schrärzende Gebilde sichtbar; ob diese Markbestandtheilen entsprechen, was nach unseren späteren Ausführungen nicht auszuschliessen ist, oder vielleicht verfetteten, zelligen Elementen, wollen wir dahin gestellt sein lassen.

Ausser diesen durch die Injection als Gefässe gekemnzeichneten Gehilden fand ich nun eigenartige, breite blasse Fasern, die zweifellos Nervenfasern sind (vgl. Fig. 4 ans dem peripheren Stumpf des Ischiadicus eines 4 Wochen alten Thieres 6 Wochen nach der Durchschneidung). Es sind dies keine Gefässe, da einerseits von der Injectionsmasse nie etwas sichtbar war, andererseits die Fasern in ihrem Verlaufe Abschnitte zeigten, die sie zweifellos als nervöse Gebilde documentirten. Solche auffallenden breiten Fasern, wie sie Fig. 4 wiedergiebt, entsprechen wohl einem Theil der von Gudden beschriebenen Gebilde; mit letztgenanntem Autor bin ich der Ansicht, dass es sich bier höchstwahrscheinlich um degenerirte, aber schon in Regeneration begriffene Nervenfasern handelt; jedoch wage ich nicht zu entscheiden, ob dieselben besonders mit Rücksicht auf ihre Breite eine eigenartige Form des. Umwandlungsprocesses darstellen, oder ob wir es hier mit einem unentwirrbaren Convolut einzelner Fasern zu thun haben, dier uns solche auffallenden Bilder geben.

Jedenfalls aber haben wir hier zweifellos Nervenfasern vor uns. So sehen wir denn in Fig. $4 \mathrm{im}$ oberen Theile massenhafte Anhäufung von Markklumpen, durch die die Schwan'sche Scheide förmlich auf- 
getrieben erscheint, sodam im unteren Abschnitte, welcher, was liarbe, Breite und Configuration betrifft, durchaus an die vorhin beschriebenen Gebilde erinnert, die längsovalen oder spindelförmigen gewucherten Kerne der Sehw ann'schen Sebeide, die $z$. Th. noch gröbere Markkügelchen enthalten, zumeist aber nur eine ganz feine Körnung zeigen. Nach den Arbeiten von Stroebe (Ziegler, Beiträge, Bd. XIIl) und Stransky (Journal für Psychologie und Neurologie 1903) u. A. ist man wohl berechtigt, jenen gewucherten Kernen die Rolle von Phagocyten zuzuschreiben, die das zerfallende Mark wegtransportiren. Stroebe beobachtet (l. c.) dreierlei Zustände an der Nervenfaser:

1. leere Abschnitte mit stellenweise fadenförmigen, collabirten Schwann'schen Scheiden.

2. Faserpartieen, ausgefüllt mit gewucherten myelinfreien Neurilemmzellen; ihre Kerne sind längsoval und liegen parallel der Faser; ihr Protoplasma fïllt in Spindelform eine Strecke der Nervenröhre aus.

3. Myelinhaltige Stellen von grossem Querschnitt, darin noch frei Nyelintrümmer, oder sie sind schon in die zu Phagocyten gewordenen gewucherten Zellen der Schwann'schen Scheide eingelagert.

Sodaun sagt er: „Die mit Spindelzellen erfüllten Abschnitte nehmen immer grössere Strecken in Besitz, so dass sie wie breite Züge parallel laufender, sehr langer Spindelzellen aussehen. Die freien Myelintrümmer treten gegen die intracellulären immer mehr zurück".

Zu dieser dritten Kategorie gehören Fasern, wie sie Fig. 4 darstellt und diesen sehen die fraglichen, sich theilenden Fasergebilde z. Th. so ähnlich (vgl. die Abbildungen), dass nur die Injectionen mit der Farbflüssigkeit die wesentliche und generelle Verschiedenheit anfdeckten. Freilich kommen auch noch andere Bilder of genug zur Beobachtung, die sicb schwer oder gar nicht den bekannten an die Seite stellen lassen. Hier will ich auch gleich bemerken, dass ich so bizarre Formen, wie sie Gudden (l. c.) in Fig. 26 a und 27 a und c giebt, nicht fand; häufig dagegen sah ich Bilder, die seinen Figuren 19 bis 24 auffallend gleichen.

Wie kommen also auf Grund unserer Untersuchungen zu dem Resultate, dass höchstwahrscheinlich im peripheren Nervensystem eigenartige, breite, blasse, jedenfalls in Regeneration begriffene Nervenfasern. wie Gudden es annahm, vorkommen, an denen wir jedoch niemals, wie dieser Autor, Theilungen wahrnehmen konnten. Alle sich theilenden Gebilde sind vielmehr nach unseren Befunden Gefässe, und es wirft sich die Frage auf, ob und was für besondere Verhältnisse hier vorliegen, die zu ihrer Auffassung als Nervenfasern führem konnten.

Gudden hebt hervor, dass er die eigenartigen Fasem bei normalen 
Individuen und gesuuden Kaninchen nicht gefunden habe. Hierin decken sich meine Befunde mit denen Gudden's, da ich diese Fasern in den resecirten Nervenstücken, die mir als Controlpräparate dienten, ebenso wenig in den Fällen von Nephritis u. a., die keine Nervenerkrankung klinisch gezeigt hatten, finden konnte: jedenfalls fand ich keine so typischen Bilder, wie in den peripheren, zur Degeneration gebrachten Nervenstümpfen; in den centralen Strimpfen waren sie auch schon seltener, aber doch einige Male vorhanden.

Wie ist dies Verhalten zu erklären? Stroebe sagt l. c.: „Gleichzeitig mit den ausserhalb der alten Neurilemmschläuche gelegenen Phagocytenansammlungen sehen wir solche in der 4. und 5. Woche anch noch an einem anderen Orte, nämlich in der adventitiellen Lymphscheide der Blutgefässe des peripheren Nervenabschnittes, vor Allem der kleinen Arterien. Die kleinen Gefässe scheinen von einem manchmal ziemlich breiten Saum der mit Myelintropfen beladenen Phagocyten umgeben.... Die adventitiellen Lymphscheiden vieler im Imern der peripheren Nervenstrecken verlaufenden Blutgefässe sind mit Zellen, welche den Phagocyten genau gleichen, dicht erfüllt, so dass jetzt diese Gefässe genau dasselbe Aussehen bieten, wie ein mit einem Saume von Körnchenzellen umgebenes Blutgefäss in der Nähe eines cerebralen Degenerationsherdes".

Stroebe hat sich der Anilinblau-Saffraninfärbonng bedient und seine Aufmerksamkeit auf die Gefässe bei Osmiumzupfpräparaten nicht gelenkt, hat daher dieselben. Bilder wie Gudden und ich nicht zu Gesicht bekommen.

Auch Büngner (Ziegler, Beitr. Bd. X.) spricht von der Be. theiligung der Gefässe bei dem Proliferationsprocess im peripheren Nervenstumpf: „An den bindegewebigen Hüllen der Nerven und den Endothelkernen der Gefässe sind Kerntheilungsprocesse erkennbar, wenn auch nicht so stark hervortretend".

Ferner wird bei E. Remak (Neuritis und Polyneuritis. 1900) der Proliferationsvorgänge der Kerne der Schwann'schen Scheide sowohl wie der Endothelien der Blutgefässe und der Zellen des Perineuriums gedacht.

Hiernach ist es wun garnicht mehr auffullig, dass wir bei normalen Nerven solehe Nervenfasern vortäuschenden Gefässe im Allgemeinen nicht finden, dagegen wach Resection von Nervenstückchen in peripheren Stümpfen, in denen der ganze Nerv mit allen seinen Gebilden eine lebhafte Umwandlung erfährt. An dem Umwandlungsprocess im peripheren Nervenstumpf betheiligen sich also dessen Gefässe sebr wesentlich und zeigen uns streckenweise oft Bilder, zum Theil durchaus 
ähnlich denen der in Regeneration begriffenen Nervenfasern. Wir sehen nämlich sowohl Nervenfasern wie kleine Blutgefässe mit den Phagocyten besetzt; letztere enthalten hier wie dort kleine Markanhäufungen, zeigen dieselbe Anordnung in Reihen, dieselbe Grösse und Gestalt, so dass beide Fasergebilde dadurch einander sehr ähnlich werden, vergl. z. B. Fig. 2 und 4. Auch Gudden's Abbildungen der breiten, blasseu Fasern lassen Bildungen, die offenbar diesen Phagocyten entsprechen, sowohl an den von ihm beschriebenen, sich theilenden, wie den sich nicht theilenden Fasern erkennen.

Noch eines Punktes möchte ich im Folgenden kurz Erwähnung thun, der zwar nicht in den Rahmen dieser Arbeit gehört, jedoch mein Intcresse erregte, da A. Westphal in seiner Arbeit (Die elektrischen Erregbarkeitsverhältnisse des peripheren Nervensystems des Menschen im jugendlichen Zustande und ihre Beziehung zum anatomischen Bau derselben. Bd. XXVI d. Areh.) dazu anffordert, die Verhältnisse der Markscheidenbildung nach experimentellen Eingriffen und die in der normalen Entwickelung begründeten in vergleichender Weise festzustellen.

Durch die Liebenswürdigkeit meines Chefs war es mir gestattet, die von $i h m$ angefertigten Nervenzupfpräparate Neugeborener zu studiren. Aus den Resultaten seiner Arbeit ]. c. hebe ich folgende Punkte hervor: Bei den Nervenfasern Neugeborener ist in quantitativer Hinsicht bedeutend weniger Mark vorhanden, die Markscheiden sind viel dünner, zeigen oft Unterbrechungen von beträchtlicher Länge; die Yarkablagerung ist eine unregelmässige. Osmium färbt einen grossen Theil des peripheren Nervenmarks Neugeborener grünlicli oder graugelblich; eine schwärzliche Färbung dagegen nehmen nur relativ spärliche Fasern an; die unentwickelten Fasern lassen keine Ranvier'schen oder Lantermannschen Einschnürungen erkennen. Die Kerne der Schwann'schen Scheide sind auffallend gross, ron ausgedebnten protoplasmatischen Massen umgeben und anscheinend zahlreicher als beim Erwachsenen.

Die sich neu regenerirenden Nervenfasern bei jugendlichen Thieren gleichen nun auch nach meinen Befunden (vergl. Fig. 9 u. 10) in auffallender Weise dieser Beschreibung und den Abbildungen A. West phal's, eine Thatsache, die dieser Autor mit Hinweis auf die bekannten Untersuchungen Erb's bereits hervorgehoben hat. Die degenerirten Fasern kehren also bei der Regeneration gleichsam in den embryonalen Zustand zurück, in welchem eine Scheidung zwischen Mark und Axencylinder noch nicht besteht. (A. Westphal l. c.)

Aus meinen Untersuchungen möchte ich noch hinzufügen, dass ich in dem resecirten Stück des Ischiadicus eines 5 Tage alten Kaninchens die meisten Fasern schon mit ganz zarter Markscheide versehen fand; 
einzelne jedoch zeigten stellenweise sich neubildendes Mark, stellenweise waren sie noch marklos (Fig. 12). Jedenfalls sahen diese feinen dünnen Fasem ebenfalls hellgränlich aus und boten durchaus ähnliche Bilder wie die der Neugeborenen. Nebenbei bemerkt sei noch, dass ich bei diesen ganz jungen Thieren im resecirten Stück auffallender Weise, allerdings ganz vereinzelt, degenerirte Fasem mit ganz schwarzen Markbröckeln fand.

Nach 6 bis 7 Wochen sah ich bei denselben nach Resection des Ischiadicus im peripheren Stumpf keine Fasern, die das Bild des gewöhnlichen degenerativen Zerfalls darboten, sondern nur ganz schmale, wellig verlaufende Fäserchen (Fig. 9 n. 10), die in ziemlich regel mässigen Intervallen Anschwellungen zeigten. Bei Saffraninfärbung noch deutlicher als bei Osmiumzupfpräparaten liessen sich diese spitzovalen Gebilde als im Verlaufe der Faser liegend, erkemmen. In Uebereinstimmung mit A. Westphal (l. c. S. 82, vergl. Fig" 4-6 daselbst) handelt es sich hier höchstwahrscheinlich um beginnende Markbildung in der Umgebung der grossen Kerne der Schwan'schen Scheide. Dafür spricht bei Osmiumzupfpräparaten auch die intensivere Färbung der gedachten Anschwellungen. Wir sehen hier also im peripheren Stumpf übcrall den regenerativen Process Platz greifen, während von Iregeneration nichts mehr erkennbar ist.

Bei älteren Thieren von 5 Wochen bis $3 / 4$ Jahr dagegen finden sich 6-7 Wochen nach der Durchschneidung des Nerven im peripheren Stumpf degenerirte Fasern vom gewöhnlichen Typus des groben Markzerfalles (Fig. 11).

Darnach dürfte wohl an die Möglichkeit zu denken.sein, dass, wie überhaupt bei jungen Thieren die Lebensenergie eine grössere ist (vergl. Neumann, Arch. f. mikr. Anat. Bd. XVIII.), so auch die Energie des regenerativen Processes im peripheren Nervensystem sich gesteigert zeigen kann ${ }^{1}$ ). So wären dieser Annahme gemäss die verschiedenen Bilder, die man trotz gleicher Zeit nach der Excision bei verschiedenen Thieren erhält, zı erklären, wenn man deren Alter berücksichtigt.

Von grossem Interesse wäre es, dieser Frage an der Hand eines grösseren Materials weiter experimentell nachzugehen.

Fasse ich zum Schluss kurz das Resultat meiner Befunde zusammen, so ergiebt sich:

1) Anm. Wir glauben nicht, dass diese Annahme sich mit den fundamentalen Thatsachen der B. v. Gudden'schen Atrophie der Nervenelemente bei Neugeborenen, deren Eigenheit in einem weit tiefer greifenden und. schnelleren Verlauf der Atrophie als bei. Erwachsenen besteht, im Widerspruch befindet. 
1. Die von Gudden beschriebenen breiten blassen Fasem sind, soweit sie Theilungen aufweisen, kleinste Gefässe, allerdings in verz̈ndertem Zustande, indem an denselben sich Zellen, die morphologiseh den gewucherten Zellen der Schwann'schen Scheide (Phagocyten) durchaus ähnlich sind, ansammeln und wohl dadurch die eigenartigen Bilder bedingen.

2. Es kommen bei degenerativen Processen im peripheren Nervensystem Bilder zu Gesicht, die bis auf die feblende Theilung den breiten blassen Fasern Guddens entsprechen, und die zweifellos Nervenfasern darstellen. Wenn wir anch in Uebereinstimmung mit $\mathrm{Gudden}$ die Anuahme, dass es sich um in Regeneration begriffene Nervenfasern handelt, für die wahrscheinlichste halten, so möehtẹn wir doch nicht zu entscheiden wagen, ob wir es hier mit einer besonderen Form des regenerativen Processes zu thun haben, oder ob diese eigenartigen Bilder durch ein Convolut einzelner Fasern vorgetäuscht werden.

3. Die in Entwickelung begriffenen Nervenfasern wenige T'age alter Thiere (Kaninchen) sind durchaus äbulich denen, die mehrere Wochen nach der Durchschneidung des Nerven im peripheren Stumpf desselben Thieres sich in der Regeneration befinden.

4. In Osminmzupfpräparaten von Fasern aus den peripheren Nervenstümpfen, die bei verschieden alten Thieren zu derselben Zeit angelegt sind, findet man 6-7 Wochen nach der Durchschneidung des Nerven bei älteren Thieren eclatante Degenerationserscheinungen, während bei ganz jungen Individuen in derselben Zeit schon durchweg die Restitution Platz gegriffen hat.

Am Schlusse meiner Abhandlung erfülle ich die angenehme Pficht, meinem hochverehrten Chef, Herrn Prof Dr. A. Westphal, für die Anregung zu vorliegendem Thema sowohl wie für die überaus liebenswürdige Belehrung und Unterstützung bei der Arbeit meinen ergebensten Dank auszusprechen.

\section{Erklärung der Abbildungen (Taf. X).}

Alle Fasern sind unter $1 / 12$ Oel-Imm. (Hartnack) gezeichnet.

Fig. 1. Faser aus dem Mcdianns einer 32 jähr. an Inanition zu Grunde gegangenen Melancholica. Osmiumzupfpräparat. Mit grösster Wahrscheinlichkeit ein Gefäss. Text S. 492.

Fig. 2. Faser aus dem Peroneus eines Paralytikers. Text S. 493. kennbar.

Fig. 2a. Dieselbe Faser an anderer Stelle; hier dentlich als Gefäss er- 
Fig. 3. Faser aus dem Peroneus einer an Dementia senilis zu Grunde gegangenen Frau; es bestanden deutliche neuritische Erscheinungen. In Osmiumpräparaten fanden sich ziemlich häufig degenerirte Nervenfasern mit bröckeligem Markzerfall.

Fig. 3a. Dieselbe Faser an anderer Stelle; sich theilend und in der äussern Form veränderte Blutkörperchen enthaltend.

Fig. 4. Nerrenfaser aus dem peripheren Stumpf eines 5 Wochen alten Kaninchens 6 Wochen nach der Durchschneidung. Im oberen Theil grosse Markballen, die die Schwann'sche Scheide stark ausdehnen; unterhalb der Knickstelle kleinere Marktrümmer z. Th. in wuchernden spitz ovalen Gebilden (Phagocyten) liegend, welche eine feine Körnelung aufweisen.

Fig. 5. Ein ähnlich aussehendes Gebilde von demselben Thier; Gefäss; central die blane Injectionsmasse, rechts unten Ansatz zur Teilung.

Fig. 6, 7 und 8. Fasern aus dem peripheren Nervenstumpf eines $3 / 4$ Jahr alten Thieres, das 7 Wochen nach der Durchschneidung des $N$. ischiadicus ausserordentlich starke Degenerationserscheinungen darbot. In Fig. 6 oben die injicirte Masse, in der Mitte schwache Markpartikelchen (verfettete Endothelien?).

Fig. 9. Saffraninpräparat aus dem peripheren Stumpfe des N. ischiadicus eines Thieres, dem im Alter von 5 Tagen ein Stück dieses Nerven resecirt wurde, 6 Wochen nach der Operation. In dem peripheren Stumpf waren keine degenerativen Erscheinungen mehr sichtbar, sondern überall Restitution.

Fig. 10. Dasselbe bei Osmiumfärbung.

Fig. 11. Faser aus dem peripheren Nervenstumpf eines ca. 12 Wochen alten Thieres. In dem ebenfalls 6 Wochen alten peripheren Stumpfe steht überall der degenerative Process im Vordergrund. In dem unteren Theil der Faser wohl schon beginnende Regeneration.

Fig. 12. Faser aus dem resecirten Stück des Ischiadicus eines 5 Tage alten Thieres: normale junge sich entwickelnde Faser mit Markanhäufungen in ziemlich gleichmässigen Intervallen (Osmiumzupfpräparat, in welchem der Achsencylinder nicht distinkt gefärbt ist). 
\&े

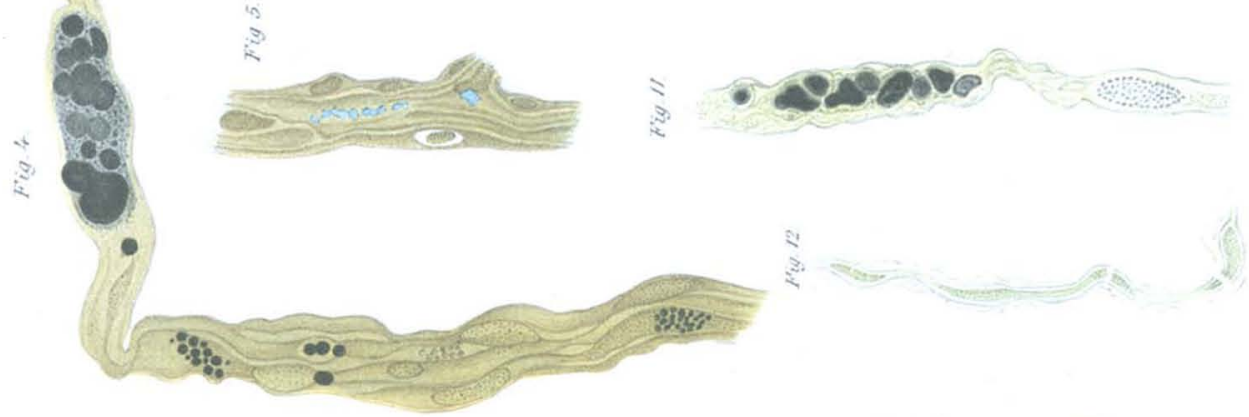

$3 \frac{0.0000}{0-0.005}$
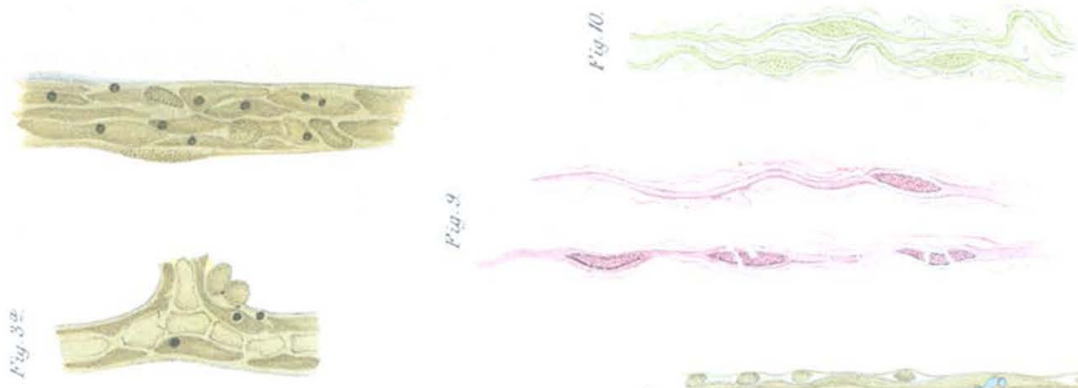

30.00 .00 .00

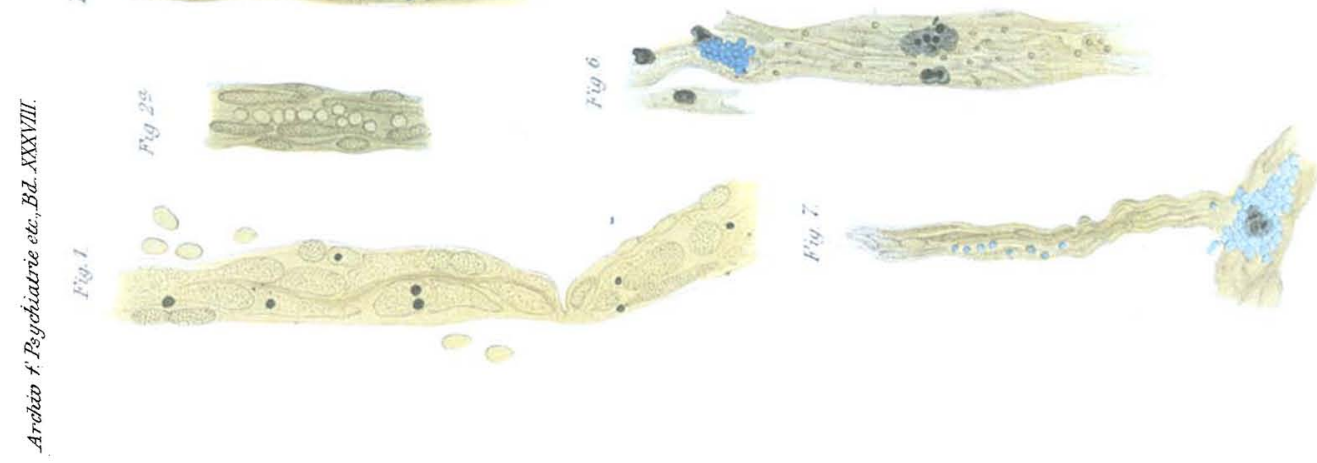

